\title{
Corrigendum
}

\section{Compactifications of $\mathbb{C}^{3}$. II}

\section{Thomas Peternell}

Mathematisches Institut, Universität Bayreuth, D-95440 Bayreuth, Germany

Math. Ann. 283, 121-137 (1989)

Received June 29, 1993

In the paper [Pe] I studied algebraic compactifications $X$ of $\mathbb{C}^{3}$ with second Betti number 1 (which are necessarily Fano manifolds) and non-normal divisor at infinity $Y$. The main result was the following:

Theorem. If the index of $X$ is 1 (see [Pe] for this and the following notions), then its genus is 12 , in particular $X$ is rational with $b_{3}=0$.

In the proof however there is a gap as pointed out by Furushima and consequently some of the cases of the finer classification (structure of $Y$ ) are missing. The aim of this note is to show how to complete the proof. The main result, together even with very nice effective constructions has been proved independently by Furushima [Fu].

In the following I am using all the notations of [Pe]. The trouble arises in Proposition 3.6 where the following is claimed

$\tilde{E}$ is non-reduced iff $H^{1}\left({ }_{\mathrm{red}} \tilde{E}\right)=0$.

The proof made use of $H^{0}(\psi) \neq 0$ which is not necessarily the case. Therefore the case

(A) $H^{0}(\psi)=0$ and $\tilde{E}$ non-reduced

has to be treated separately. Since (3.6) has been used for parts of (3.7) and also the map $\phi$ constructed in (3.7) need not to be surjective (which is unrelated to the failure of 3.6), we have another case to be treated

(B) $H^{0}(\psi) \neq 0$ and $\tilde{E}$ is non-reduced.

Therefore Proposition 3.6 and 3.7 have to be read as follows.

Proposition 3.6. Assume $H^{0}(\psi) \neq 0$. Then $\tilde{E}$ is non-reduced iff $H^{1}\left(\mathcal{G}_{\mathrm{red}} \tilde{E}\right)=0$.

Proposition 3.7. Let $\tilde{E}_{j}$ be a non-reduced component of $\tilde{E}$ such that red $\tilde{E}_{j}$ is smooth. Then, letting $\mathscr{T}$ be the ideal sheaf of $\operatorname{red} \tilde{E}$ in $\tilde{E}$, the sheaf $\left(\mathscr{F} / \mathscr{T}^{2}\right) \mid \operatorname{red} \tilde{E}_{j}$ divided by its torsion is either 0 or its dual contains a trivial subsheaf.

This form of (3.7) is sufficient for all applications. The proofs of (3.6) and (3.7) are given in $[\mathrm{Pe}]$, in fact, this is what is really proved there. 
Let us now give the proof of the theorem in the missing cases. We begin treating case (A). We proceed in three steps.

(1) Because of our assumptions we have

$$
H^{1}\left(\bigodot_{\mathrm{red} \tilde{E}}\right)=\mathrm{C}, \quad H^{1}(\tilde{E}, \mathbb{Z})=0 .
$$

Hence red $\tilde{E}$ consists of three smooth rational curves passing through one point plus some extra trees of rational curves. This is an easy exercise on curves using the techniques of $[\mathrm{Pe}]$.

(2) These extra trees are immediately shown to be non-existent using the information $\omega_{\tilde{E}} \cong \mathcal{E}_{\tilde{E}}$. So $\tilde{E}$ consists of three components. Let $r$ be the number of components of $E$, clearly $r \leqq 2$. If $r=2$, then red $E$ consists of 2 smooth rational curves meeting transversely in one point, otherwise of one smooth rational curve.

(3) Using the topological sequence [Ba-Ka, 3.A.7, (3.8)], we conclude $b_{3}(Y) \leqq$ $b_{3}(\tilde{Y})+2$. Since $b_{3}(Y)=b_{3}(X)$ and $b_{3}(\tilde{Y})=b_{3}$ of a desingularisation are both even, we have either $b_{3}(Y)=b_{3}(\tilde{Y})$, then we are done by the same arguments as in [Pe, p. 132-134], or $b_{3}(Y)=b_{3}(\tilde{Y})+2$, then these arguments need only slightly to be modified.

The case $r=2$ can actually be eliminated as by similar computations as presented in $(B)$.

We now treat (B). So $\tilde{E}$ is non-reduced and $H^{0}(\psi) \neq 0$, so $H^{1}\left(\bigodot_{\operatorname{red}} \tilde{E}\right)=0$. In particular red $\tilde{E}$ is a tree of $\tilde{r}$ smooth rational curves. We want to prove $\tilde{r}=1$, then all the arguments of [Pe] go through. So assume $\tilde{r} \geqq 2$ and let $A$ be a non-reduced component of $\tilde{E}$ with reduction $A_{0}$. Using (2.3) and (2.4) of [Pe] for $A_{0} \subset A \subset \tilde{E}$ we obtain

$$
H^{0}\left(\omega_{A}\right)=0
$$

and consequently

$$
\operatorname{Hom}_{C_{A}}\left(n, \omega_{A}\right)=0
$$

where $n$ is the sheaf of nilpotent functions on $A$. In order to contradict (1) it is sufficient to exhibit $n$ as subsheaf of $\omega_{A}$ which we are going to do now. $\omega_{A}$ is nothing but the ideal sheaf of the analytic intersection $A \cap B$ in $A$ where $B$ is the union of the other components of $\tilde{E}$. It is sufficient to show that $A \cap B$ is reduced. Observe that $\omega_{A}$ is in a canonical way a subsheaf of $\omega_{\tilde{E}} \mid A$, since $\omega_{A}=$ Hom $_{\tilde{E}}\left(\bigodot_{A}, \bigodot_{\tilde{E}}\right)$, similarly for $\omega_{\tilde{E}_{1}}$; call the corresponding injection $f$. Assume that $A \cap B$ is not reduced. Then $\omega_{\tilde{E}} \mid A$ (modulo torsion perhaps) has degree at most -2 . Then $f$ must be isomorphic and the degree is actually -2 . In the notation of (3.7) of [Pe] this implies $\phi$ isomorphic, hence the arguments of (3.7) apply and so $\tilde{E}$ is reduced, contradiction.

This completes the proof of the main theorem of [Pe]. Also we get candidates for the non-normal locus $E$ and its preimage $\tilde{E}$. For the existence we refer to [Fu].

\section{References}

[Ba-Ka] Barthel, G., Kaup, L.: Topologie des espaces complexes compactes singulieres. Montreal Lecture Notes 80 (1982)

[Fu] Furushima, M.: The complete classification of compactifications of $\mathrm{C}^{3}$ which are projective manifolds with the second Betti number one. Preprint 1992

[Pr] Peternell, T.: Compactifications of $\mathbb{C}^{3}$. II. Math. Ann. 283, 121-137 (1989) 